\title{
Cytoplasmic Male Sterility in Russian Dandelion
}

\author{
Katrina J.M. Hodgson-Kratky and David J. Wolyn ${ }^{1}$ \\ Department of Plant Agriculture, University of Guelph, 50 Stone Road East, Guelph, Ontario, Canada \\ $N 1 G 2 W 1$
}

\begin{abstract}
Additional INDEX words. restorer of fertility, pollen, natural rubber, partial sterility, temperature-sensitive, hybrid production, Taraxacum kok-saghyz

Abstract. Russian dandelion (Taraxacum kok-saghyz) is a candidate species for introducing natural rubber production into North America, and its domestication is currently underway to create an economically viable crop. Development of hybrid cultivars is essential to maximize the yield potential of the species, and cytoplasmic male sterility (CMS) is an important breeding tool that can facilitate this process. Male sterility was discovered in two fullsib russian dandelion families, and additional crosses were performed to create $F_{2}, F_{3}$, and backcross generations to assess inheritance of the phenotype. A sterility-inducing cytoplasm (S) that could be restored to fertility by a dominant allele at a single nuclear restorer of fertility (Rf) gene and a fertility-inducing cytoplasm (F) were identified. Characteristics of the CMS system were 1) (S)rfrf $\times($ F $) r f r f$ crosses produced only sterile progeny, 2) crosses where the female parents were (F) produced only fertile progeny, and 3) segregation was observed only when the maternal parent had (S). Sterility was not stable in all plants; some with sterility-inducing cytoplasm produced small amounts of pollen or formed both sterile and fertile flowers simultaneously. On the basis of seasonal differences in the frequency of partially sterile plants, sterility was stable at low temperatures and unstable at high temperatures. This germplasm can be of central importance for development of stable CMS lines for hybrid production.
\end{abstract}

Russian dandelion is a sexually reproducing diploid $(2 n=$ $2 x=16$ ) species (Warmke, 1943) of the Asteraceae family (Whaley and Bowen, 1947), native to southeastern Kazakhstan (Hellier, 2011). Cross-pollination is enforced by a self-incompatibility (SI) system (Warmke, 1943), which is likely controlled sporophytically because this mechanism has been identified in other species within the family (Brennan et al., 2011; Hiscock, 2000). The dandelion produces large amounts of high-quality rubber in its roots (Whaley and Bowen, 1947) and is adapted to temperate climates, making it a promising candidate rubber crop for southern Canada and the northern United States. Limited breeding has been conducted with this species, so development of an economically viable crop requires improvement of many traits, such as rubber yield and plant vigor.

Male sterility is characterized by a lack of functional pollen and has been identified in over 600 species including many agriculturally important plants such as maize (Zea mays), rice (Oryza sativa), canola (Brassica napus), and sorghum (Sorghum bicolor) (Kaul, 1988). It can be inherited nuclearly, in genic male sterility (GMS), or maternally, through the mitochondrial genome, in CMS. Mutations in genes involved in anther and microspore development are the primary cause of GMS (Wilson and Zhang, 2009; Zhang and Wilson, 2009; Zhang et al., 2011). CMS genes are often created by nonhomologous recombination events in the mitochondrial genome and can contain fragments of several mitochondrial open reading frames (Horn et al., 2014). Their functions are not well understood, but they are generally thought to cause gamete abortion through disruption of mitochondrial activity or aberrant programmed cell death (Chen and Liu, 2014). However, some genes produced homeotic phenotypes, where stamens are

Received for publication 23 July 2015. Accepted for publication 4 Sept. 2015. Funding was provided by the Ontario Ministry of Agriculture and Food.

This paper is a portion of a thesis submitted by K. Hodgson-Kratky in partial fulfillment of requirements for the degree of master of science.

${ }^{1}$ Corresponding author. E-mail: dwolyn@uoguelph.ca. replaced by other reproductive organs, suggesting the cytoplasm may affect expression of nuclear genes (Linke et al., 2003).

Interactions between nuclear and mitochondrial genomes typically determine whether a plant is male sterile or male fertile. Nuclear $R f$ loci have coevolved with CMS genes and often encode RNA-binding proteins that target CMS transcripts for degradation, therefore masking the effects of the sterilityinducing cytoplasm (Chen and Liu, 2014).

Male sterility is a valuable tool for studying the mechanisms involved in anther development and gametogenesis. However, CMS most importantly can be used to facilitate hybrid seed production, which gives rise to plants with increased vigor and up to $50 \%$ greater yield compared with open-pollinated cultivars (Tester and Langridge, 2010). Both sterility (S)- and fertility (F)-inducing cytoplasms are required. Generally, a three-line system is used where a male sterile A line (S)rfrf is inbred with an isonuclear, fertile, B line (F)rfrf. After sufficient inbreeding, the A line is crossed to a genetically distinct, fertile inbred line, R. In species, such as canola, where the seed is the valuable product, the $\mathrm{R}$ line must be homozygous dominant at the $R f$ locus, $(\mathrm{F} / \mathrm{S}) R f R f$, to restore fertility to the hybrid cultivar. In russian dandelion, however, cultivar fertility is not important because the root is the harvested portion. Therefore, a commercial hybrid can be produced by crossing an (F)rfrf genotype with the A line.

GMS, in contrast, is not practical for hybridization because sterility is not maintained during inbred development (Chen and Liu, 2014). GMS that is affected by climatic conditions, termed environment-sensitive GMS (EGMS), however, can be used for hybrid production because the male sterile line can be inbred under conditions inducing fertility and then crossed under conditions inducing sterility. Photoperiod and temperature-sensitive GMS lines account for $\approx 20 \%$ of seed production in China (Huang et al., 2014).

Male sterility was discovered previously, in France, in diploid Taraxacum species belonging to the Erythrosperma 
Table 1 . Genetic analysis of russian dandelion $\mathrm{F}_{1}$ progeny, testing cytoplasmic inheritance of male sterility and nuclear inheritance of a restorer of fertility $(R f)$ gene.

\begin{tabular}{|c|c|c|c|c|c|c|}
\hline \multirow[b]{2}{*}{ Model $^{z}$} & \multirow[b]{2}{*}{ Cross no. } & \multirow[b]{2}{*}{ Cross } & \multicolumn{2}{|c|}{ Segregation ratio $(\mathrm{F}: \mathrm{S})^{\mathrm{y}}$} & \multirow[b]{2}{*}{$\chi^{2 \mathrm{x}}$} & \multirow[b]{2}{*}{$P$} \\
\hline & & & Observed & Expected & & \\
\hline$(\mathrm{S}) R f r f \times(\mathrm{F}) r f r f=1(\mathrm{~S}) R f r f: 1(\mathrm{~S}) r f r f$ & 1 & Fertile- $1 \times$ Fertile- 2 & $38: 35$ & $1: 1$ & 0.05 & 0.82 \\
\hline & 2 & Fertile- $3 \times$ Fertile- 4 & $19: 15$ & & 0.26 & 0.61 \\
\hline
\end{tabular}

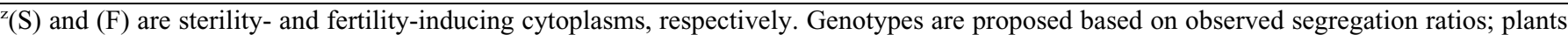
with (S)rfrf genotype are sterile and plants with genotypes (S)Rfrf, (F)Rfrf, and (F)rfrf are fertile.

${ }^{\mathrm{y}}$ Fertile and sterile phenotypes are represented by $\mathrm{F}$ and $\mathrm{S}$, respectively.

${ }^{x}$ Observed segregation ratios were compared with expected ratios using the chi-square statistic adjusted with Yate's correction for continuity and significance was determined at $P \leq 0.05$.

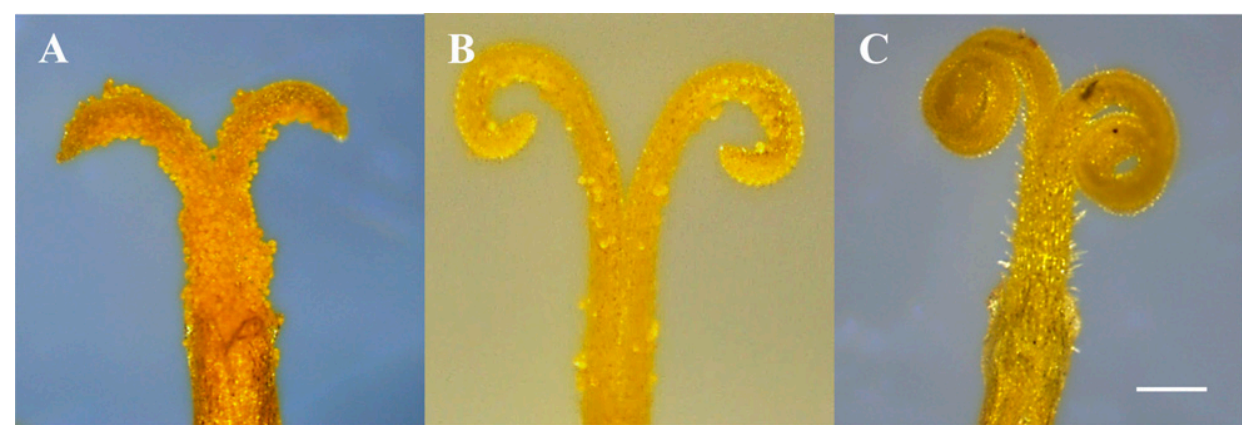

Fig. 1. (A) Male fertile, (B) partially sterile, and (C) sterile russian dandelion florets; scale bar $=25 \mu \mathrm{m}$.
Crosses were then performed among phenotypes and families to produce $\mathrm{F}_{2}$ progeny for analysis. $\mathrm{F}_{2}$ plants were intermated and backcrossed to the parents to create the $\mathrm{F}_{3}$ and $\mathrm{BC}_{1}$ families, respectively. Up to 10 flower heads per plant were observed in the $\mathrm{F}_{2}, \mathrm{~F}_{3}$, and $\mathrm{BC}_{1}$ generations to accurately identify plants as sterile, partially sterile, and fertile (Fig. 1). Partially sterile plants produced small amounts of pollen on some or all inflorescences, and/or produced both completely

and Ruderalia sections of the Asteraceae (van der Hulst et al., 2004). Genetic analyses with male fertile diploid and triploid plants indicated that the sterility was inherited cytoplasmically and dominant alleles at two $R f$ loci could restore fertility. Russian dandelion is classified in the Macrocornuta section of the Asteraceae (Richards, 1968); therefore, newly discovered male sterility in this species could have evolved independently from the germplasm discovered in France. The trait has also been identified in Taraxacum gilliesii, native to South America; however, inheritance is undetermined (Uhlemann et al., 2004).

The objective of this experiment was to determine the inheritance of the male sterile phenotype identified in two full-sib russian dandelion families. Crosses were performed to create $\mathrm{F}_{2}, \mathrm{~F}_{3}$, and backcross $\left(\mathrm{BC}_{1}\right)$ generations. Analysis of segregation ratios indicated that the sterility-inducing factor was cytoplasmically inherited and presence of a dominant $R f$ allele could restore fertility.

\section{Materials and Methods}

Genetic Materials and analysis. Populations consisting of the PIs W6 35156, W6 35159, W6 35160, W6 35162, W6 35164, W6 35165, W6 35166, W6 35168, W6 35169, W6 35170, W6 35172, W6 35173, W6 35176, W6 35177, W6 35178, W6 35179, W6 35180, W6 35181, W6 35182, and W6 35183 (Washington State University Regional Plant Introduction Station, Pullman, WA) were developed and individual crosses were performed. Details for the collection of PIs in Kazakhstan are described by Hellier (2011).

Male sterility was observed segregating in two reciprocal crosses (Table 1). One flower head from each plant of the two crosses was observed visually and phenotyped as sterile or fertile, based on the absence or presence of pollen, respectively. fertile and sterile capitula (flower heads). To determine the genetic model, partially sterile plants were grouped in the sterile phenotypic class because they likely resulted from leakiness of the sterile mutation.

GROWTH AND CULTURE. For each generation of genetic analysis, seeds were germinated in petri dishes with moistened filter paper. Seedlings were transplanted into 50-cell plug trays filled with peat-based medium (Sunshine LC1; Sun Gro Horticulture, Vancouver, BC, Canada) on 8 Oct. 2013, 9 Apr. 2014, and 19 Aug. 2014 for $F_{1}, F_{2}$, and $F_{3}$ plantings, respectively. Backcrosses were planted with the $\mathrm{F}_{2}$, and residual seeds from some $\mathrm{F}_{2}$ families were replanted for evaluation with $\mathrm{F}_{3}$ seed to increase sample size and assess the effect of season on stability of the male sterile phenotype. Some $F_{2}$ families were also evaluated initially with the $\mathrm{F}_{3}$.

Seedlings were repotted $30 \mathrm{~d}$ after planting into $12.7-\mathrm{cm}-$ diameter pots filled with peat-based medium (Sunshine LC1). All plants were grown in a greenhouse with a 16 -h photoperiod, using natural light supplemented with a photosynthetic photon flux of $50-70 \mu \mathrm{mol} \cdot \mathrm{m}^{-2} \cdot \mathrm{s}^{-1}$ produced by high-pressure sodium lamps. Phenotypic evaluation occurred from 20 Nov. 2013 to 4 Jan. 2014, 14 May 2014 to 10 July 2014, and 21 Sept. 2014 to 20 Nov. 2014 for $F_{1}, F_{2}$, and $F_{3}$, respectively. Air temperature was set to $21 / 18{ }^{\circ} \mathrm{C}$ (day/night), but averaged $24 / 20{ }^{\circ} \mathrm{C}$, and fluctuated between 20.7 and $33.8^{\circ} \mathrm{C}$ day and 18.2 and $24.9^{\circ} \mathrm{C}$ night from April to mid-September, during evaluation of the $\mathrm{F}_{2}$. Plants were fertigated on alternate weeks with $20 \mathrm{~N}-3.5 \mathrm{P}-16.6 \mathrm{~K}$ plus micronutrients (all purpose high $\mathrm{N}$ fertilizer; Plant Products, Leamington, ON, Canada) at a concentration of $1.5 \mathrm{~g} \cdot \mathrm{L}^{-1}$.

Crossing Methods. Plants were individually bagged with open-ended $33.0 \times 40.7-\mathrm{cm}$ polypropylene microperforated bags (Elkay Plastic Co., Los Angeles, CA). Tops of bags were open to reduce condensation, which caused buds to rot. 


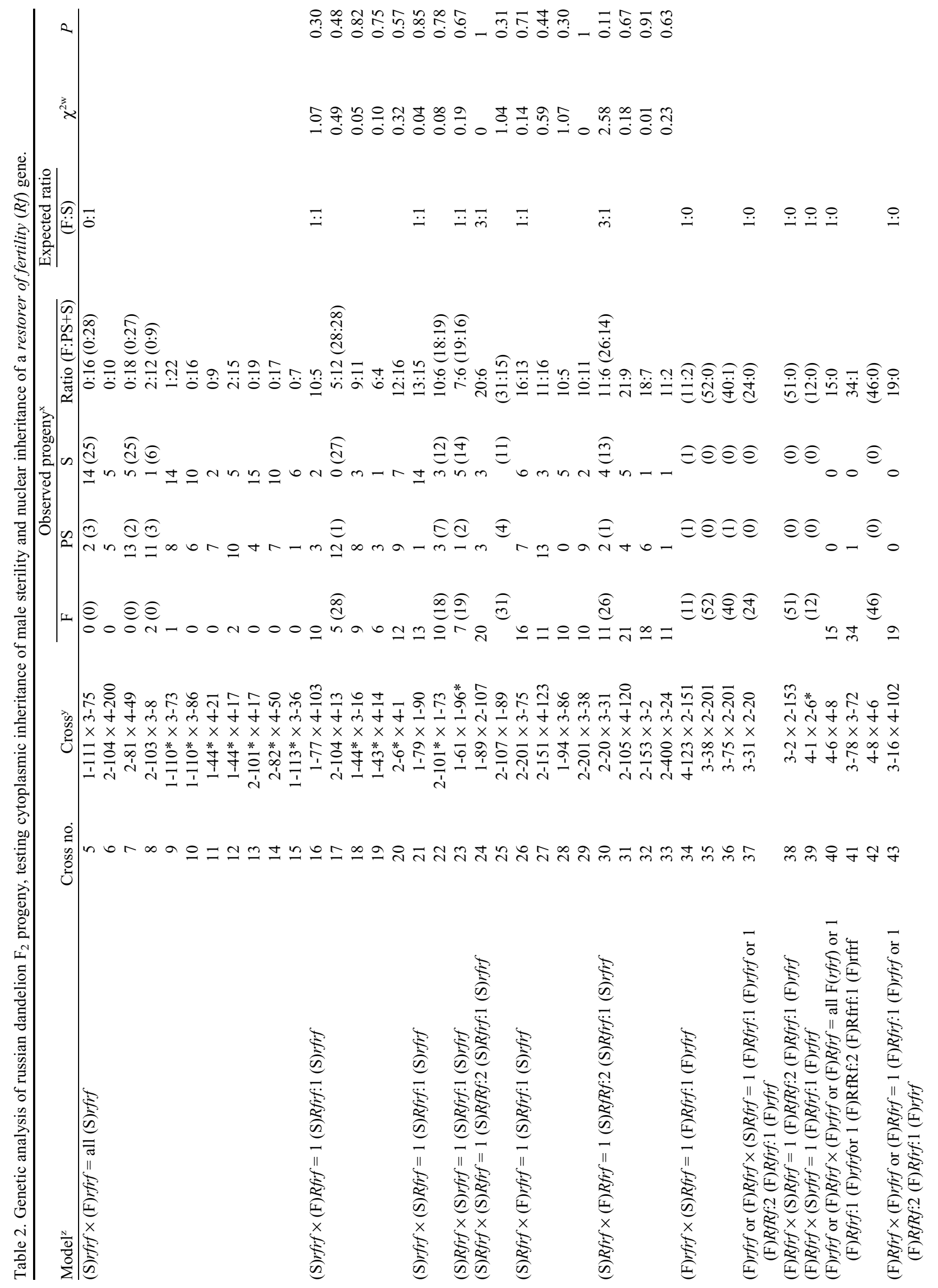




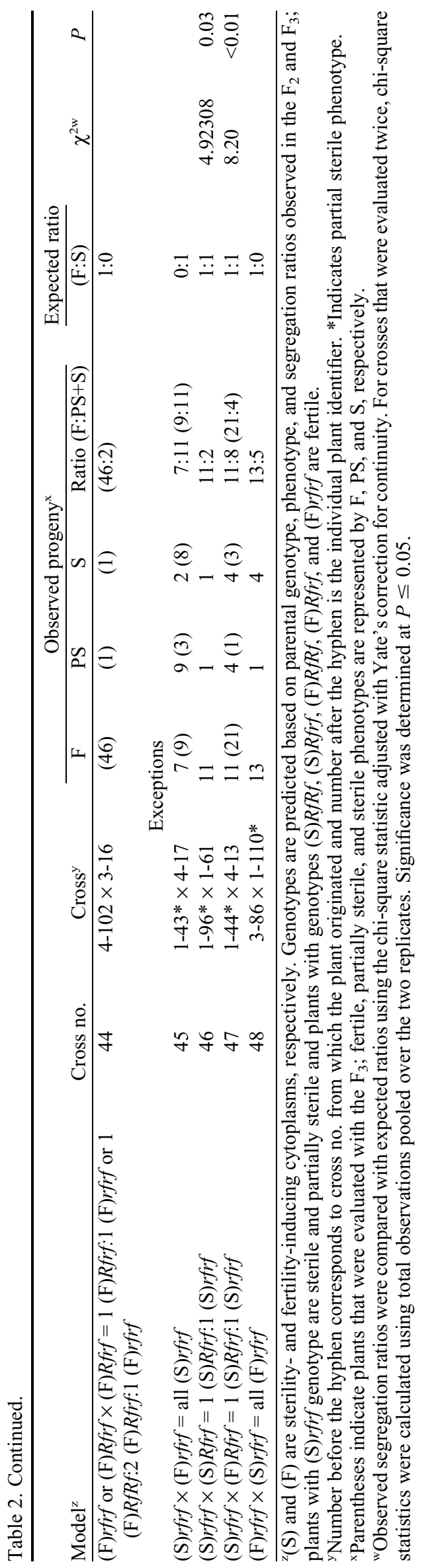

Pollination was accomplished by rubbing capitula together. No action was taken to prevent self-pollination because russian dandelion is self-incompatible. However, unpollinated capitula of bagged plants were observed to ensure self-pollination was not occurring or greenhouse insects were not affecting pollination. These capitula contained aborted seed indicating that self-fertilization and cross-contamination through insect vectors was not an issue.

Statistical analyses. Observed and expected segregation ratios were compared using the Pearson goodness-of-fit test, estimated with the PROC FREQ procedure in SAS (version 9.3; SAS Institute, Cary, NC). Since there were two phenotypic classes, the chi square analysis was adjusted with Yate's correction for continuity (Bowley, 2008). Heterogeneity tests were used for $F_{2}$ crosses that were planted on each of two planting dates, to determine whether segregation was significantly different (Mather, 1952). Since all crosses had homogeneous replicates, the chi square statistic was calculated using total observations pooled across the two plantings. Significance for all chi square tests was determined at $P \leq 0.05$. The percentage of partially sterile plants was calculated.

\section{Results}

$\mathbf{F}_{1}$. Reciprocal differences were observed when two pairs of male fertile plants were crossed; families segregated 1 male sterile: 1 fertile, or all fertile (Table 1). Intercrossing progeny from these two crosses to produce the $\mathrm{F}_{2}$ (Table 2) resulted in development of a model for the original crosses, where plants with a sterility-inducing cytoplasm (S) and heterozygous for a nuclear restorer, $R f r f$, where the dominant allele restores fertility, were crossed to plants with fertility-inducing cytoplasm (F) and homozygous recessive for restorer, rfrf. The observed segregation (Table 1) could be explained as:

$$
\begin{aligned}
& \text { A: }(\mathrm{S}) R f r f \times(\mathrm{F}) r f r f=1(\mathrm{~S}) \operatorname{Rfrf}(\text { fertile }): 1(\mathrm{~S}) r f r f(\text { sterile }) \\
& \text { B: }(\mathrm{F}) r f r f \times(\mathrm{S}) R f r f=1(\mathrm{~F}) \operatorname{Rfr}(\text { fertile }): 1(\mathrm{~F}) r f r f(\text { fertile })
\end{aligned}
$$

$\mathbf{F}_{2}$. Crossing the sterile progeny from A (above) with fertile progeny from $\mathrm{B}$, resulted in two classes of segregation; one had only sterile progeny as predicted for a $(\mathrm{S}) r f r f \times(\mathrm{F}) r f r f$ cross [crosses 5-15 (Table 2)] and the second segregated 1 fertile: 1 sterile as predicted for a (S)rfrf $\times(\mathrm{F}) R f r f$ cross (crosses 16$20)$. For a few crosses $(8,9$, and 12$)$, one or two fertile plants were observed in families that were mostly sterile. These were considered to be contaminants or phenotyped incorrectly due to environmentally sensitive CMS expressivity (i.e., they had partial sterile phenotypes but conditions favored production of fully fertile capitula). Intermating sterile and fertile progeny predicted from A, $(\mathrm{S}) r f r f \times(\mathrm{S}) R f r f$ produced 1 fertile $(\mathrm{S}) R f r f$ : 1 sterile (S)rfrf segregation (crosses 21-23). A cross between parents with the (S)Rfrf genotype produced the expected 3:1 segregation (crosses 24 and 25). When plants with the (S)Rfrf genotype were also crossed as females to plants with (F) cytoplasm, progeny segregated either 1:1 (crosses 26-29) or 3:1 (crosses 30-33), which is consistent with the presumed genotypes of (F)rfrf and (F)Rfrf for the pollen donors, respectively. Reciprocals of these crosses and all other crosses where the female parent had (F) cytoplasm (crosses 34-44) yielded all fertile progeny further confirming the genotypes of these plants. 
Many plants $[3-75,2-104,1-110,3-86,1-44,4-17,4-13$, 3-16, 1-43, 2-6, 4-1, 2-201, 1-114, and 3-78 (Table 2)] were used as parents in multiple crosses to validate their genotypes, and in most cases, the proposed genotype was correct for each cross. For example, 3-75 was identified as (F)rfrf because only sterile progeny were produced when it was crossed to a sterile plant [cross 5 (Table 2)]. It was then crossed reciprocally to a fertile plant, (S)Rfrf, and progeny segregated 1:1 (cross 26) and 1:0 (cross 36) as predicted.

Residual seeds of the $F_{2}$ that were planted simultaneously with the $\mathrm{F}_{3}$ had the same phenotypic segregation ratios (Table 2) as proven by heterogeneity tests $[P>0.08$ (data not shown)] indicating that season did not affect segregation. Of the families that were planted twice, the percentage of plants classified as partially sterile was $60 \%$ during the first evaluation in the summer and $15 \%$ when replanted seeds were evaluated in late autumn when average temperatures were $24 / 20$ and $21 / 18{ }^{\circ} \mathrm{C}$ (day/night), respectively.

BACKCROSSES. Backcrosses of segregating progeny to the original parents also supported the genetic model. Crossing a (S) rfrf plant to the (S)Rfrf and (F)rfrf parents resulted in 1:1 segregation (cross 49) and all sterile progeny (cross 50), respectively (Table 3). Crosses between segregating plants with (F) cytoplasm and the (S)Rfrf parent produced only fertile progeny (crosses 51 and 52) and 3:1 segregation (cross 53), when the original parent was used as the female and male, respectively.

$\mathbf{F}_{3}$. The segregating families of the $\mathrm{F}_{3}$ further confirmed the genetic model. Crossing two partially sterile plants, (S)rfrf, produced only sterile progeny [cross 55 (Table 4)]. Sterile plants, (S)rfrf, crossed to those with (F) cytoplasm and unknown restorer alleles resulted in only fertile (cross 56) or only sterile (cross 57) progeny indicating the male parents were (F) $R f R f$ and (F)rfrf, respectively. Cross 58 also produced only sterile progeny as predicted. Crosses between sterile (S)rfrf and fertile (S)Rfrf plants produced progeny segregating $1: 1$, as expected (crosses 59-62).
Intercrossing fertile plants with $(\mathrm{S})$ cytoplasm produced both 3:1 [crosses 63-66 (Table 4)] and 1:0 (crosses 67 and 68) segregation ratios, as expected, for crosses $(\mathrm{S}) R f r f \times(\mathrm{S}) R f r f$ and $(\mathrm{S}) R f R f \times(\mathrm{S}) R f r f$, respectively. Plants with (F) cytoplasm, when crossed as females to both fertile (crosses 69-71) and partially sterile (cross 72) plants, produced only fertile progeny. Fertile plants, (S)Rfrf crossed to (F)rfrf genotypes, segregated 1:1 (crosses 73-75).

Similar to the $\mathrm{F}_{2}$, many parents from the $\mathrm{F}_{3}[22-80,43-49$, 13-100, 24-9, 31-13, 21-4, and 47-7 (Table 4)] were used in multiple crosses, and proposed genotypes were validated, thus affirming the genetic model. Also, multiple plants originating from specific $\mathrm{F}_{2}$ families were used as parents to produce the $\mathrm{F}_{3}$, and the expected genotypes were identified among siblings. For example, crosses 58,69, 70, 74, and 75, used different siblings from cross 48 (Table 2), and confirmed (F)rfrf was the only genotype among the fertile $\mathrm{F}_{2}$ progeny. Parental genotypes for cross 24 (Table 2) were predicted to be $(\mathrm{S}) R f r f \times(\mathrm{S}) R f r f$ with fertile progeny segregating as (S)RfRf and (S)Rfrf; both progeny genotypes were validated in crosses $59,65-68$, and 75 (Table 4).

The identification of (S) cytoplasm and $R f$ alleles in two independent crosses (Table 1) could be explained by one common or two genetically distinct mechanisms to induce sterility and restore fertility. Intercrossing fertile and sterile progeny from the two original reciprocal crosses [crosses 16, 19 , and 22 (Table 2)] resulted in 1:1 segregation, suggesting the two sources of $R f$ alleles can restore both sources of cytoplasm and the presence of only one genetic class of (S) cytoplasm. Crossing heterozygous plants containing dominant restorer alleles from each source (cross 24) produced progeny segregating 3:1, with predicted genotypes: (S)RfRf, (S) $R f r f$, and (S)rfrf. Only fertile progeny were recovered when intercrossing fertile offspring from cross 24 [crosses 67 and 68 (Table 4)], which is expected for a cross between (S)RfRf and (S)Rfrf. If there were two $R f$ genes, segregation ratios of $3: 1$,

Table 3. Genetic analysis of russian dandelion backcross $\left(\mathrm{BC}_{1}\right)$ progeny, testing cytoplasmic inheritance of male sterility and nuclear inheritance of a restorer of fertility $(R f)$ gene.

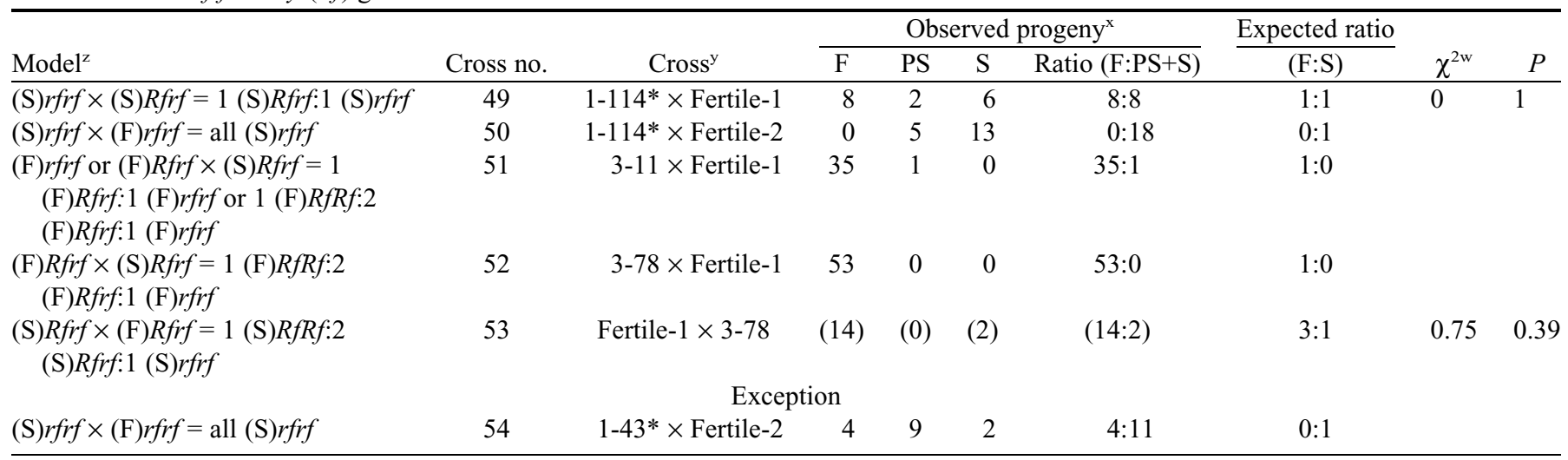

$\overline{{ }^{z}}(\mathrm{~S})$ and $(\mathrm{F})$ are sterility- and fertility-inducing cytoplasms, respectively. Genotypes are predicted based on parental genotype, phenotype, and segregation ratios observed in $\mathrm{BC}_{1}$ progeny; plants with (S)rfrf genotype are sterile and partially sterile and plants with genotypes ( $\left.\mathrm{S}\right) R f R f$, (S)Rfrf, (F)RfRf, (F)Rfrf, and (F)rfrf are fertile.

${ }^{\mathrm{y}}$ Number before the hyphen corresponds to cross no. from which the plant originated and number after the hyphen is the individual plant identifier. *Indicates partial sterile phenotype.

${ }^{x}$ Parentheses indicate plants that were evaluated with the $\mathrm{F}_{3}$; fertile, partially sterile, and sterile phenotypes are represented by $\mathrm{F}$, PS, and $\mathrm{S}$, respectively.

wObserved segregation ratios were compared with expected ratios using the chi-square statistic adjusted with Yate's correction for continuity. Significance was determined at $P \leq 0.05$. 
Table 4. Genetic analysis of russian dandelion $\mathrm{F}_{3}$ progeny, testing cytoplasmic inheritance of male sterility and nuclear inheritance of a restorer of fertility $(R f)$ gene.

\begin{tabular}{|c|c|c|c|c|c|c|c|c|c|}
\hline \multirow[b]{2}{*}{ Model $^{\mathrm{z}}$} & \multirow[b]{2}{*}{ Cross no. } & \multirow[b]{2}{*}{ Cross $^{\mathrm{y}}$} & \multicolumn{4}{|c|}{ Observed progeny ${ }^{\mathrm{x}}$} & \multirow{2}{*}{$\frac{\text { Expected ratio }}{(\mathrm{F}: \mathrm{S})}$} & \multirow[b]{2}{*}{$\chi^{2 \mathrm{w}}$} & \multirow[b]{2}{*}{$P$} \\
\hline & & & $\bar{F}$ & PS & $\mathrm{S}$ & Ratio (F:PS+S) & & & \\
\hline$(\mathrm{S}) r f r f \times(\mathrm{F}) R f R f=$ all $(\mathrm{S}) R f r f$ & 56 & $9-25^{*} \times 40-920$ & 11 & 0 & 0 & $11: 0$ & $1: 0$ & & \\
\hline & 58 & $13-100 \times 48-3$ & 0 & 1 & 25 & $0: 26$ & & & \\
\hline \multirow[t]{2}{*}{$(\mathrm{S}) r f r f \times(\mathrm{S}) R f r f=1(\mathrm{~S}) R f r f: 1(\mathrm{~S}) r f r f$} & 59 & $5-13 \times 24-9$ & 9 & 1 & 13 & $9: 14$ & $1: 1$ & 0.70 & 0.40 \\
\hline & 60 & $13-100 \times 31-13$ & 18 & 7 & 4 & $18: 11$ & & 1.24 & 0.27 \\
\hline$(\mathrm{S}) R f r f \times(\mathrm{S}) R f r f=1(\mathrm{~S}) R f R f: 2$ & 63 & $21-4 \times 21-35$ & 12 & 0 & 2 & $12: 2$ & $3: 1$ & 0.38 & 0.54 \\
\hline \multirow[t]{3}{*}{ (S)Rfrf:1 (S)rfrf } & 64 & $47-7 \times 21-4$ & 20 & 0 & 5 & $20: 5$ & & 0.12 & 0.73 \\
\hline & 65 & $24-9 \times 31-10$ & 32 & 7 & 3 & $32: 10$ & & 0 & 1 \\
\hline & 66 & $31-10 \times 24-9$ & 50 & 7 & 8 & $50: 15$ & & 0.05 & 0.83 \\
\hline$(\mathrm{S}) R f r f \times(\mathrm{S}) R f R f=1(\mathrm{~S}) R f R f: 1(\mathrm{~S}) R f r f$ & 67 & $24-9 \times 24-8$ & 34 & 0 & 0 & $34: 0$ & $1: 0$ & & \\
\hline$(\mathrm{F}) R f R f \times(\mathrm{S}) \mathrm{r} f r f=$ all $(\mathrm{F}) R f r f$ & 72 & $40-920 \times 9-25^{*}$ & 42 & 0 & 0 & $42: 0$ & $1: 0$ & & \\
\hline \multirow[t]{3}{*}{$(\mathrm{S}) R f r f \times(\mathrm{F}) r f r f=1(\mathrm{~S}) R f r f: 1(\mathrm{~S}) r f r f$} & 73 & $47-7 \times 43-49$ & 19 & 2 & 18 & $19: 20$ & $1: 1$ & 0 & 1 \\
\hline & 74 & $21-4 \times 48-20$ & 9 & 0 & 6 & $9: 6$ & & 0.27 & 0.61 \\
\hline & 75 & $24-9 \times 48-899$ & 17 & 1 & 8 & $17: 9$ & & 1.88 & 0.17 \\
\hline & & Excepti & & & & & & & \\
\hline \multirow[t]{2}{*}{$(\mathrm{S}) r f r f \times(\mathrm{S}) R f r f=1(\mathrm{~S}) R f r f: 1(\mathrm{~S}) r f r f$} & 76 & $18-1000 \times 23-20$ & 57 & 0 & 0 & 57:0 & $1: 1$ & 55.02 & $<0.01$ \\
\hline & 77 & $22-80 \times 23-12$ & 21 & 0 & 8 & $21: 8$ & & 4.97 & 0.03 \\
\hline
\end{tabular}

$\overline{\mathrm{z}}(\mathrm{S})$ and $(\mathrm{F})$ are sterility- and fertility-inducting cytoplasms, respectively. Genotypes are predicted based on parental genotype, phenotype, and segregation ratios; plants with (S)rfrf genotype are sterile and partially sterile and plants with genotypes (S)RfRf, (S)Rfrf, (F)RfRf, (F)Rfrf, and (F)rfrf are fertile.

${ }^{y}$ Number before the hyphen corresponds to cross no. from which the plant originated and number after the hyphen is the individual plant identifier. ${ }^{*}$ Indicates partially sterile phenotype.

${ }^{x}$ Fertile, partially sterile, and sterile phenotypes are represented by F, PS, and S, respectively.

${ }^{w}$ Observed segregation ratios were compared with expected ratios using the chi square statistic adjusted with Yate's correction for continuity.

Significance was determined at $P \leq 0.05$.

$7: 1$, or $15: 1$ would be expected; thus only one $R f$ gene is interacting with the $(\mathrm{S})$ cytoplasm.

Exceptional Crosses. A small number of crosses did not support the genetic models (Tables 2-4). These results may be explained by misphenotyping due to weak penetrance of the $R f$ allele, experimental error, or presence of additional segregating genes.

\section{Discussion}

A source of CMS, restored to fertility by an $R f$ allele at a single locus, has been identified in russian dandelion. The model is validated by the recovery of: 1) only sterile progeny in crosses identified as (S)rfrf $\times(\mathrm{F}) r f r f, 2)$ only fertile progeny when plants with (F) cytoplasm were used as females, and 3) 1:1 and 3:1 segregation only when plants with (S) cytoplasm were used as females. The consistency and predictability of segregation over three generations of crosses further demonstrated the genetic model. Although van der Hulst et al. (2004) also identified CMS in Taraxacum species collected in France, the germplasm did not include russian dandelion. Genetic and molecular analyses are necessary to determine the relationship between the mechanisms of CMS and fertility restoration in the different species.

Some plants were classified as partially sterile because they produced both sterile and fertile flower heads and/or reduced pollen on some or all capitula. It was assumed that these plants were unstable sterile plants with genotype (S) rfrf, and that individual genetic background, environment, and genotype $\times$ environment interactions (Weider et al., 2009) caused this phenotypic variability. Partial sterility can also be explained in a limited number of crosses as poor penetrance of the restorer allele in the $(\mathrm{S}) R f$ genotype. In 18 of 23 crosses, progeny from partially sterile parents segregated as predicted, indicating that the proposed genotype for these plants was correct. Interestingly, all exceptional crosses in Table 2 that did not fit predicted ratios included a partially sterile parent.

Some $\mathrm{F}_{2}$ families were planted and evaluated twice, first during the summer and then in late autumn. All families had consistent segregation of fertile and sterile plants indicating that season did not affect ratios; however, the percentages of sterile plants that were unstable (partially sterile) when evaluated in the summer and autumn were 60 and 15, respectively. This difference may be explained by seasonal environmental variation. Greenhouse temperature was variable but averaged $24 / 20{ }^{\circ} \mathrm{C}$ (day/night) during the summer evaluation, while stable temperatures of $21 / 18{ }^{\circ} \mathrm{C}$ were maintained in the autumn. Consequently high temperature may have caused a partial reversion to fertility in plants with the (S) cytoplasm. Similarly, van der 
Hulst et al. (2004) found fertility reversion in male sterile dandelions, which they attributed to temperature. However, temperature had the opposite effect to that reported here: partial sterility was observed at low temperatures, $\leq 15^{\circ} \mathrm{C}$, but plants were sterile when grown above $17^{\circ} \mathrm{C}$. In rice, sterility can be induced in different thermo- and photoperiod-sensitive GMS lines by either high or low temperature or long or short photoperiod, respectively (Peng et al., 2008).

Associations between temperature and CMS stability have also been determined in several species including pigeon pea [Cajanus cajan (Ariyanayagam et al., 1995)], cotton [Gossypium arboreum (Sarvella, 1966)], petunia [Petunia $\times$ hybrida (van Marrewijk, 1969)], and maize (Weider et al., 2009). In canola, plants with (S) cytoplasm were sterile when grown at a temperature of $22 / 16^{\circ} \mathrm{C}$ (day/night) but partially and fully reverted to fertile when grown at 26/20 and 30/24 ${ }^{\circ} \mathrm{C}$, respectively (Fan and Stefansson, 1986). Minor seasonal changes for other environmental conditions that are known to affect CMS expression such as photoperiod (Murai and Tsunewaki, 1993), humidity (Ariyanayagam et al., 1995), and total solar radiation (Sarvella, 1966) and their interactions with individual genotypes may also have influenced expressivity in russian dandelion.

Temperature-sensitive CMS is not practical for hybrid seed production because purity can be compromised by selfing during unfavorable conditions. The lack of partial steriles in some matings and varying levels of partial sterility among crosses suggest that nuclear genetic background could affect the trait. Consequently, one may be able to breed for stable CMS. SI has also been used as an alternative to CMS in other species as a means to generate hybrids (Kucera et al., 2006; Rahman, 2005; Watanabe and Hinata, 1999). Further analysis of SI in russian dandelion and development of a method to promote selfing for inbred development could result in efficient hybrid production.

\section{Literature Cited}

Ariyanayagam, R.P., A. Nageswar Rao, and P.P. Zaveri. 1995. Cytoplasmic-genic male sterility in interspecific matings of Cajanus. Crop Sci. 35:981-985.

Bowley, S. 2008. A hitchhiker's guide to statistics in biology. 2nd ed. Any Old Subject Books, Guelph, ON, Canada.

Brennan, A.C., D.A. Tabah, S.A. Harris, and S.J. Hiscock. 2011. Sporophytic self-incompatibility in Senecio squalidus (Asteraceae): $\mathrm{S}$ allele dominance interactions and modifiers of cross-compatibility and selfing rates. Heredity $86: 113-123$.

Chen, L. and Y. Liu. 2014. Male sterility and fertility restoration in crops. Annu. Rev. Plant Biol. 65:579-606.

Fan, Z. and B.R. Stefansson. 1986. Influence of temperature on sterility of two cytoplasmic male sterility systems in rape (Brassica napus L.). Can. J. Plant Sci. 66:221-227.

Hellier, B.C. 2011. Collecting in central Asia and the Caucasus: U.S. National Plant Germplasm System plant explorations. HortScience 46:1438-1439.

Hiscock, S.J. 2000. Genetic control of self-incompatibility in Senecio squalidus L. (Asteraceae): A successful colonizing species. Heredity 85:10-19.

Horn, R., K.J. Gupta, and N. Colombo. 2014. Mitochondrion role in molecular basis of cytoplasmic male sterility. Mitochondrion 13:198-205.
Huang, J., Z.E.H. Zhang, and Q. Shu. 2014. Workable male sterility systems for hybrid rice: Genetics, biochemistry, molecular biology, and utilization. Rice (N. Y.) 7:13-27.

Kaul, M.L.H. 1988. Male sterility in higher plants. Monogr. Theor. Appl. Genet. Vol. 10. Springer-Verlag, Berlin, Germany.

Kucera, V., V. Chytilova, M. Vyvadilova, and M. Klima. 2006. Hybrid breeding of cauliflower using self-incompatibility and cytoplasmic male sterility. HortScience 33:148-152.

Linke, B., T. Nothnagel, and T. Borner. 2003. Flower development in carrot CMS plants: Mitochondria affect the expression of MADSbox genes homologous to GLOBOSA and DEFICIENS. Plant J. 34:27-37.

Mather, K. 1952. The measurement of linkage in heredity. 2nd ed. Methuen, London, United Kingdom.

Murai, K. and K. Tsunewaki. 1993. Photoperiod-sensitive cytoplasmic male sterility in wheat with Aegilops crassa cytoplasm. Euphytica 67:41-48.

Peng, H.F., Z.F. Zhang, B. Wu, X.H. Chen, G.Q. Zhang, Z.M. Zhang, B.H. Wan, and Y.P. Lu. 2008. Molecular mapping of two reverse photoperiod-sensitive genic male sterility genes (rpms1 and rpms2) in rice (Oryza sativa L.). Theor. Appl. Genet. 118:77-83.

Rahman, M.H. 2005. Resynthesis of Brassica napus L. for selfincompatibility: Self-incompatibility reaction, inheritance and breeding potential. Plant Breed. 124:13-19.

Richards, A.J. 1968. The biosystematics of Taraxacum. PhD Thesis, Univ. Durham, Durham, UK.

Sarvella, P. 1966. Environmental influences on sterility in cytoplasmic male sterile cottons. Crop Sci. 6:361-364.

Tester, M. and P. Langridge. 2010. Breeding technologies to increase crop production in a changing world. Science 327:818-822.

Uhlemann, I., J. Kirschner, and J. Stepanek. 2004. The genus Taraxacum (Asteraceae) in the Southern Hemisphere. I. The section Antarctica Handel-Mazzetti and notes on dandelions of Australasia. Folia Geobot. 39:205-220.

van der Hulst, R.G., P. Meirmans, P.H. van Tienderen, and J.M. van Damme. 2004. Nuclear-cytoplasmic male sterility in diploid dandelions. Heredity 93:43-50.

van Marrewijk, G.A.M. 1969. Cytoplasmic male sterility in petunia. I. Restoration of fertility with special reference to the influence of environment. Euphytica 18:1-20.

Warmke, H.E. 1943. Macrosporogenesis, fertilization, and early embryology of Taraxacum kok-saghyz. Bull. Torrey Bot. Club 70:164-173.

Watanabe, M. and K. Hinata. 1999. Self-incompatibility, p. 149-183. In: C. Gomez-Campo (ed.). Biology of Brassica coenospecies. Elsevier Science, Amsterdam, The Netherlands.

Weider, C., P. Stamp, N. Christov, A. Hüsken, X. Foueillassar, K. Camp, and M. Munsch. 2009. Stability of cytoplasmic male sterility in maize under different environmental conditions. Crop Sci. 49:77-84.

Whaley, W.G. and J.S. Bowen. 1947. Russian dandelion (kok-saghyz) an emergency source of natural rubber. Misc. Publ. No. 618. U.S. Govt. Printing Office, Washington, DC.

Wilson, Z.A. and D. Zhang. 2009. From Arabidopsis to rice: Pathways in pollen development. J. Expt. Bot. 60:1479-1492.

Zhang, D., X. Luo, and L. Zhu. 2011. Cytological analysis and genetic control of rice anther development. J. Genet. Genomics 38:379-390.

Zhang, D. and Z.A. Wilson. 2009. Stamen specification and anther development in rice. Chin. Sci. Bull. 54:2342-2353. 